\section{Solitary fibrous tumour of the pleura causing bulging of the thoracic wall in a 44-year-old man}

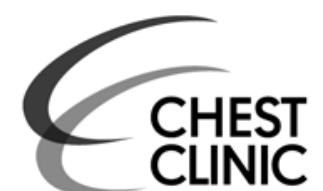

A 44-year-old male patient presented with right chest pain for 6 years, along with progressive swelling in the same region. He did not report fever, weight loss, trauma or previous surgery. On

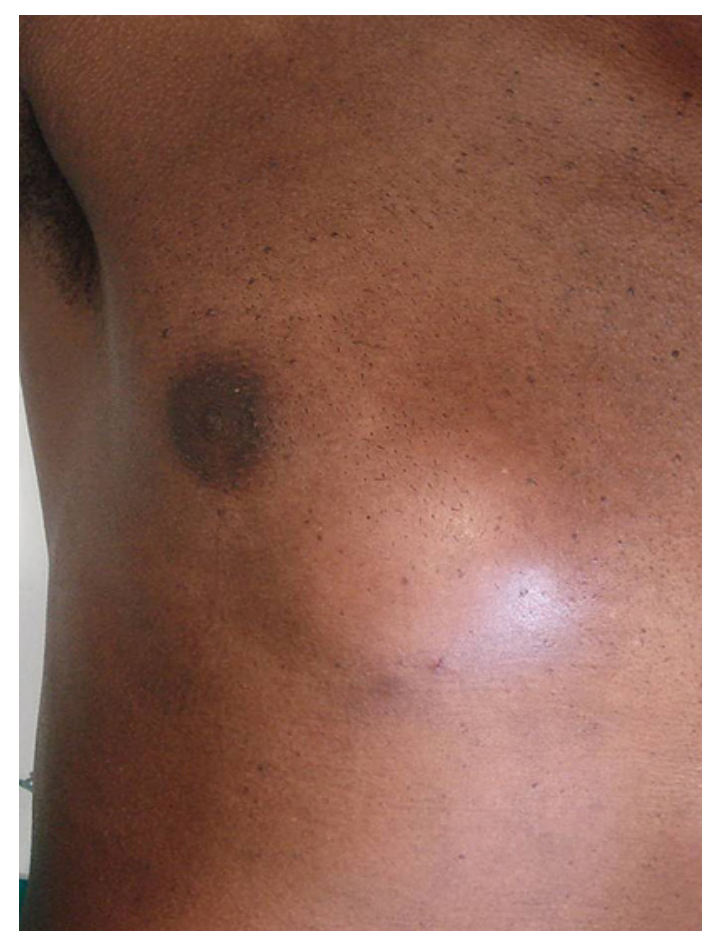

Figure 1 Focal bulging in the right hemithorax, as seen on physical examination. physical examination, a $5 \mathrm{~cm}$ focal bulge was observed in the right hemithorax just below and medial to the nipple (figure 1). Chest x-ray showed opacification of the anterior lower third of the right hemithorax (figure 2), which on CT scan corresponded to a heterogeneous mass, compressing the right lung parenchyma and the mediastinum, partially protruding through the chest wall (figure 3). These findings were confirmed by an MRI scan, which also showed that there were no signs of cardiac invasion (figure 4, online supplementary movies 1 and 2). A transparietal biopsy of the mass revealed a proliferative lesion with fusocellular areas. Immunohistochemical examination was compatible with solitary fibrous tumour of the pleura. Surgery was indicated and a heterogeneous pale mass measuring $12.5 \mathrm{~cm}$ in diameter was removed confirming the diagnosis (figure 5). Pathology showed an absence of rib or muscular infiltration, while the adjunct lung showed recent haemorrhage. Postoperative recovery was uneventful and the patient was discharged from the intensive care unit on the first postoperative day and from the hospital on the fifth postoperative day.

Solitary fibrous tumour of the pleura is an infrequent tumour (5\% of pleural neoplasms) originating from mesenchymal tissue and is benign in $80 \%$ of cases. ${ }^{1}$ It affects both sexes equally and is not related to tobacco or asbestos. Its origin may be from the visceral pleura $(80 \%)$ or from other regions with mesenchymal cells-parietal pleura, pericardium, peritoneum or the tunica albuginea. It is usually asymptomatic, but tumours larger than $10 \mathrm{~cm}$ frequently cause non-specific symptoms such as cough, dyspnoea and thoracic pain. Rarely, it causes hypoglycaemia or hypertrophic osteoarthropathy. Malignancy criteria include abundant cellularity, more than four mitoses per 10 fields at high magnification, cytonuclear atypicality, large haemorrhage/necrosis areas, pleural effusion and invasion of adjacent structures. Treatment consists of complete resection when possible, or radiotherapy/chemotherapy in cases with malignant degeneration. ${ }^{2}$

Gilberto Szarf, ${ }^{1}$ Alex Rocha Obac, ${ }^{1}$ Renato de Oliveira, ${ }^{2}$ Erica Rymkiewicz, ${ }^{2}$ Luiz Eduardo Villaca Leao, ${ }^{2}$ Henrique M Lederman'

${ }^{1}$ Department of Radiology, Federal University of Sao Paulo, Sao Paulo, Brazil; ${ }^{2}$ Department of Thoracic Surgery, Federal University of Sao Paulo, Sao Paulo, Brazil
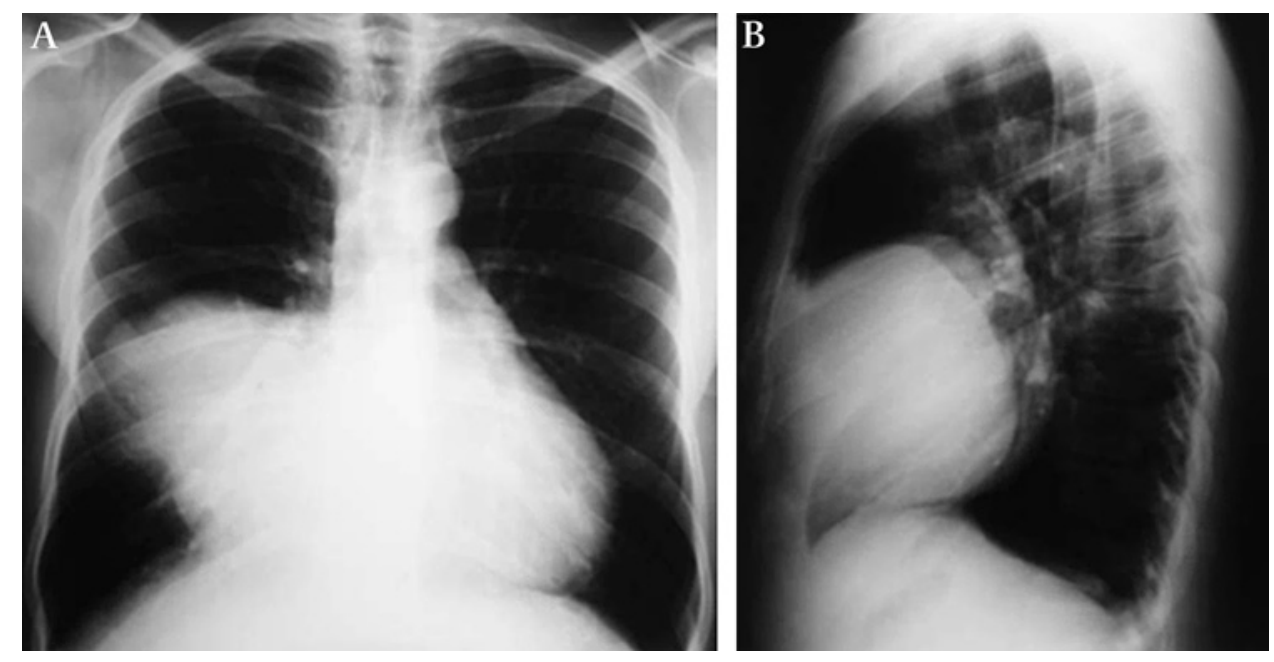

Figure 2 Chest x-ray showing opacification of the anterior lower third of the right hemithorax, with well-defined borders. 

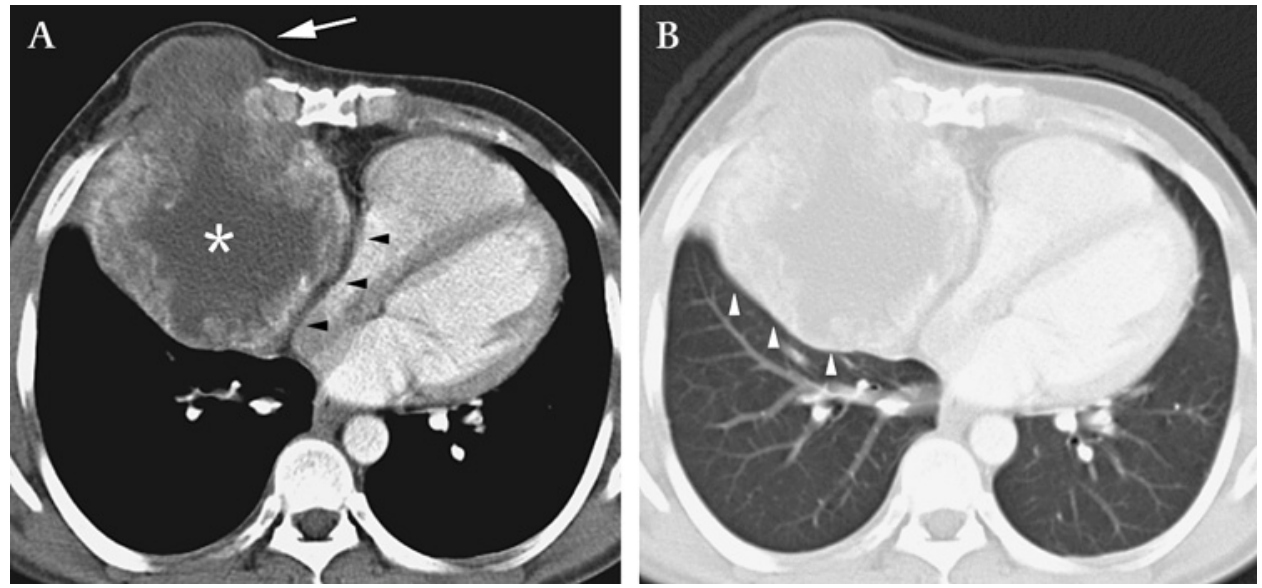

Figure 3 CT scan, $(A)$ mediastinal window and (B) lung window, demonstrating a heterogeneous mass (asterisk), compressing the right lung parenchyma (white arrowheads) and the mediastinum (black arrowheads), partially protruding through the chest wall (white arrow).
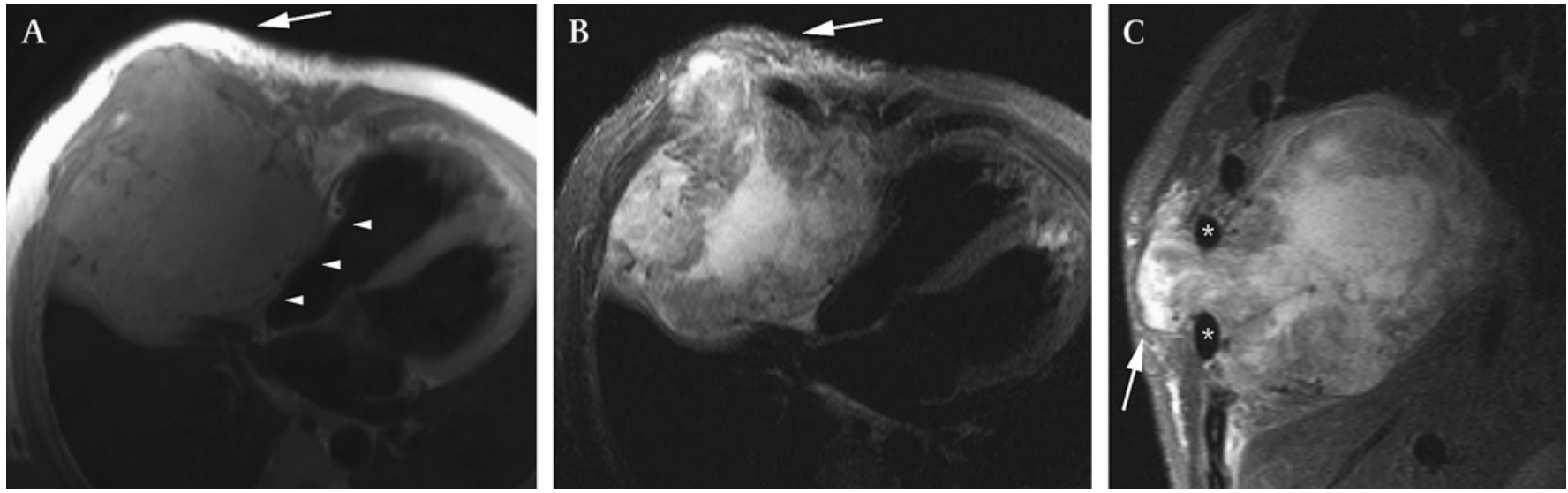

Figure 4 MR images in the (A and $B$ ) axial and $(C)$ coronal planes confirming the findings observed on $C T$, better demonstrating the protrusion of the lesion through the chest wall (white arrows), between ribs (asterisks), with no signs of cardiac invasion (white arrowheads).

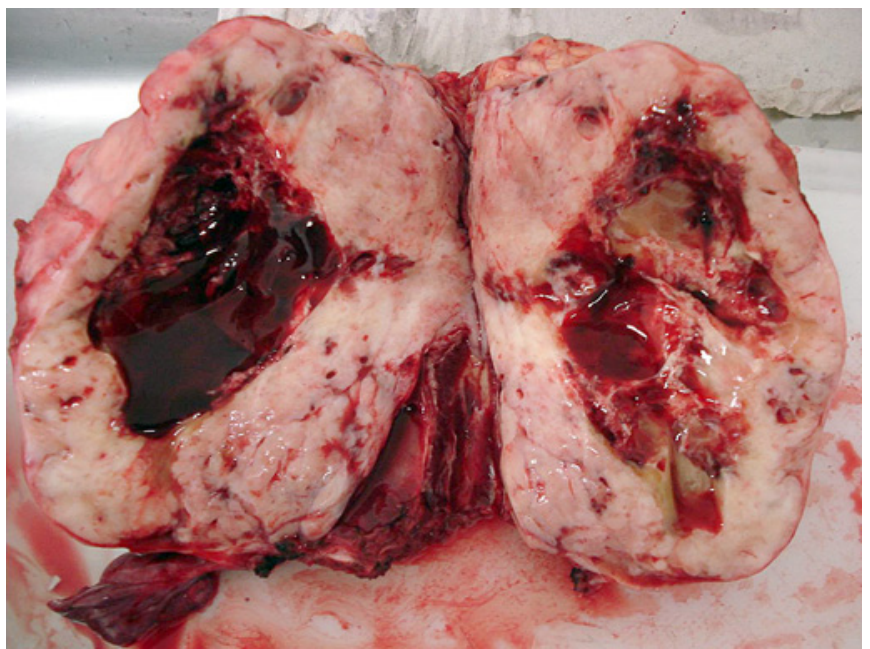

Figure 5 Pathological specimen of the resected fibrous tumour of the pleura.
Correspondence to Dr Gilberto Szarf, Rua Sergipe 605 apt. 73, Sao Paulo SP 01243-001, Brazil; gszarf@yahoo.com.br

- Additional movies are published online only. To view these files please visit the journal online (http://thorax.bmj.com/content/67/4.toc).

Acknowledgements The authors would like to thank Andrea Puchnick for editing assistance.

Competing interests None.

Patient consent Obtained.

Ethics approval This study was approved by the IRB of the Federal University of Sao Paulo.

Contributors All authors contributed extensively to the work presented in this paper.

Provenance and peer review Not commissioned; externally peer reviewed.

Received 28 June 2011

Accepted 1 August 2011

Published Online First 27 August 2011

Thorax 2012:67:374-375. doi:10.1136/thoraxjnl-2011-200701

\section{REFERENCES}

1. Weynand B, Noël H, Goncette L, et al. Solitary fibrous tumor of the pleura: a report of five cases diagnosed by transthoracic cutting needle biopsy. Chest 1997; 112:1424-8.

2. Rena 0, Filosso PL, Papalia E, et al. Solitary fibrous tumour of the pleura: surgical treatment. Eur J Cardiothorac Surg 2001;19:185-9. 